\title{
How to Break a Practical MIX and Design a New One
}

\author{
Yvo Desmedt $^{1,2 \star}$ and Kaoru Kurosawa ${ }^{3}$ \\ 1 Department of Computer Science, Florida State University \\ PO Box 4530, 206 Love Building \\ Tallahassee, FL 32306-4530, USA \\ desmedt@cs.uwm. edu \\ 2 Dept. of Mathematics, Royal Holloway \\ University of London, UK \\ 3 Dept. of Electrical and Electronic Engineering \\ Faculty of Engineering, Tokyo Institute of Technology \\ 2-12-1 O-okayama, Meguro-ku, Tokyo 152-8552, Japan \\ kurosawa@ss.titech.ac.jp
}

\begin{abstract}
A MIX net takes a list of ciphertexts $\left(c_{1}, \cdots, c_{N}\right)$ and outputs a permuted list of the plaintexts $\left(m_{1}, \cdots, m_{N}\right)$ without revealing the relationship between $\left(c_{1}, \cdots, c_{N}\right)$ and $\left(m_{1}, \cdots, m_{N}\right)$. This paper first shows that the Jakobsson's MIX net of Eurocrypt'98, which was believed to be resilient and very efficient, is broken. We next propose an efficient $t$-resilient MIX net with $O\left(t^{2}\right)$ servers in which the cost of each MIX server is $O(N)$. Two new concepts are introduced, existential-honesty and limited-open-verification. They will be useful for distributed computation in general.
\end{abstract}

\section{Introduction}

\section{$1.1 \quad$ Background}

In his extensive work to achieve anonymity, Chaum introduced the concept of a MIX net 6 . MIX nets have found many applications in anonymous communication 6, election schemes 6111924 and payment systems 14. A MIX net takes from each user a ciphertext and outputs a permuted list of the plaintexts without revealing who has sent which plaintext, i.e., which plaintext corresponds to which ciphertext. This aspect of a MIX net is also known as privacy. Although Pfitzmann-Pfitzmann 2I showed an attack against the RSA implementation of Chaum's MIX scheme, the concept itself was not broken but it was refined. The original MIX net given by Chaum 6 satisfies privacy only under the condition that all the senders are honest. To address this issue, one needs robustness. A topic that was studied prior to robustness is verifiability, to allow to detect that

\footnotetext{
* A part of this research was done while the author visited the Tokyo Institute of Technology, March 4-19, 1999. He was then at the University of Wisconsin - Milwaukee. A part of his research was funded by NSF CCR-9508528.
} 
the output of the MIX net is incorrect. If an outsider can verify this, the scheme is called universally verifiable.

Before surveying robustness and verifiability, another problem of Chaum's MIX net based on RSA should be pointed out, which is that the size of each ciphertext $c_{i}$ is long, i.e., proportional to the total number of MIX servers. Park et al. overcame this problem by using the ElGamal encryption scheme so that the size of each $c_{i}$ became independent of the number of MIX servers I9. Almost all MIX nets proposed from then on are based on the ElGamal encryption scheme.

A general method to achieving verifiability is to have each MIX server prove that it behaved correctly in zero knowledge. Sako and Kilian 24 showed such an efficient proof system for Park et al.'s MIX net. The above MIX nets are, however, not robust. If at least one MIX server stops, then the entire system stops. Ogata et al. showed the first MIX net satisfying privacy, verifiability and robustness 18 . We call a scheme satisfying all these properties resilient.

For comparison, we focus on the random permutation stage of the MIX net because almost all known resilient MIX nets consist of two stages, a random permutation stage and a threshold decryption stage. Also, there are some cases where we want a permutation, but not a decryption. If a MIX net does a permutation only, it is possible that the MIX servers do not need to know the decryption key, which is an advantage.

Now in Ogata et al.'s MIX net, the computational cost of each MIX server is $O(\kappa t N)$, where $N$ is the number of users, $\kappa$ is the security parameter and $t$ stands for the threshold number of untrusted MIX servers. Subsequently, Abe showed a more efficient resilient MIX net which is also universally verifiable in which the external verifier's cost is reduced to $O(\kappa N)$ I .

At the same time, Jakobsson showed a very efficient resilient MIX net at Eurocrypt '98 I3. (but not universally verifiable). Later, he showed a more efficient MIX net at PODC'99 15 . In these schemes, the computational cost of each MIX server is $O(t N)$.

Recently Abe $2: 3$ showed his second resilient MIX net which is efficient for a small number of users. In this MIX net, the complexity is $O(t N \log N)$. Jakobsson and Juels showed a MIX net which has the same advantage 16. In their MIX net, the cost of each MIX server is $O\left(t N \log ^{2} N\right)$. Since these complexities grow faster in $N$ than the other schemes, these schemes suit small $N$.

\subsection{Our Contribution}

This paper first shows that the Jakobsson's first MIX net (presented at Eurocrypt'98) 13, which was believed to be resilient and very efficient, is not robust. We present an attack such that at least one malicious MIX server can prevent computing the correct output. We exploit a homomorphic property of Jakobsson's Eurocrypt '98 scheme to attack it. Observe that we make no claims about other MIX networks, such as the PODC'99 Jakobsson paper 15 .

We also propose a new and very efficient resilient MIX net (but it is not universally verifiable). To obtain this scheme, we introduce three new concepts: 
Work-sharing-MIX in which (significantly) more MIX servers are being used than one trusts. In threshold schemes a tradeoff is used between reliability and privacy. The motivation of work-sharing-MIX is to have a tradeoff between the number of MIX severs and the computational effort per MIX sever. When $N$ is large (as in national elections), and one wants a sufficiently high security (i.e., a large $t$ ), then the computational effort of existing schemes may be prohibitive. We share the computational effort over several machines while maintaining the requirements as privacy, robustness and verifiability.

Existential-honesty divides the MIX servers into blocks of which we can guarantee that one is free of dishonest MIX servers, assuming the number of dishonest MIX servers is bounded by $t$.

Limited-open-verification is the opposite of zero-knowledge. To prove that a computation has been done correctly the party that did the computation in a block will open the secret it used. However, she will only open this to the members in the same block.

More details are given later on. Those concepts may be useful in other contexts such as secure distributed computation. We achieve $100 \%$ robustness in contrast with prior schemes (i.e. the probability of the failure of robustness is 0 ).

Although the total computational cost of our scheme is comparable to the one of Jakobsson's MIX net of PODC'99 I5 (i.e. $O\left(t^{2} N\right)$ ), the computational cost of each MIX server is significantly smaller (i.e. $O(N)$ ) in ours versus the one in Jakobsson's scheme $(O(t N))$. To achieve this we need $O\left(t^{2}\right)$ MIX servers rather than the usual $O(t)$. This introduces several open problems, which we discuss in Sect. 6

Other details, such as the computational complexity assumptions we need to prove privacy are discussed later on.

\section{Model of MIX Net}

\subsection{Model and Definitions}

In the model of MIX nets, there exist three types of participants: users, a bulletin board, and the MIX servers.

1. The users post encrypted messages $\left(c_{1}, \cdots, c_{N}\right)$ to the bulletin board.

2. After the bulletin board fills up, or after some other triggering event occurs, the mix servers compute a randomly permuted list of decryptions $\left(m_{1}, \cdots, m_{N}\right)$ of all valid encryptions posted on the bulletin board.

MIX nets must satisfy privacy, verifiability and robustness. Suppose that at most $t$ among $v$ MIX servers and at most $N-2$ among $N$ senders are malicious. Then we say that a MIX net satisfies :

- $t$-privacy if the relationship between $\left(c_{1}, \cdots, c_{N}\right)$ and $\left(m_{1}, \cdots, m_{N}\right)$ is kept secret. 
- $t$-verifiability if an incorrect output of the MIX net is detected with overwhelming probability.

- t-robustness if it can output $\left(m_{1}, \cdots, m_{N}\right)$ correctly with overwhelming probability.

We say that a MIX net is $t$-resilient if it satisfies $t$-privacy, $t$-verifiability and $t$-robustness.

\subsection{ElGamal Based Encryption Scheme for Users}

ElGamal based encryption scheme was commonly used in some of the previous robust MIX nets $18 \mid \mathrm{I}$. Let $p$ be a safe prime, i.e., $p, q$ be primes such that $p=2 q+1$, and $g$ be a generator of $G_{q}$. Let $y=g^{x} \bmod p$, where $x$ is a secret key. The public key is $(p, q, g, y)$.

The MIX servers share a secret key $x$ using a $(t+1, v)$ threshold scheme 27 , where $v$ denotes the number of MIX servers.

To encrypt a value $m \in G_{q}$, a random number $\gamma \in \in_{u} Z_{q}$ is chosen and the ciphertext $(a, b)=\left(g^{\gamma}, m y^{\gamma}\right)$ is calculated. For decryption, $m=b / a^{x}$ is calculated by a threshold decryption scheme 82012

As pointed out by Jakobsson, to guarantee that $m \in G_{q}$, we should let $m=(M \mid p) M$ for an original message $M \in[1 \ldots(p-1) / 2]$, where $(M \mid p)$ is the Jacobi symbol of $M$.

\section{$2.3 \quad$ Non-malleable ElGamal}

Malicious users may post copies or correlated ciphertexts of some encrypted messages of honest users (repeated ciphertext attack). They can then determine (with some probability) what the decryption of the attacked message was, by counting repeats or correlations in the output list. Therefore, it is necessary to use a non-malleable encryption scheme. A public key cryptosystem is said to be non-malleable 9 if there exists no probabilistic polynomial time (p.p.t.) adversary such that given a challenge ciphertext $c$, he can output a different ciphertext $c^{\prime}$ such that the plaintexts $m, m^{\prime}$ for $c, c^{\prime}$ are meaningfully related. (For example, $m^{\prime}=m+1$.)

Tsiounis and Yung 28, and independently Jakobsson 1.3, showed a nonmalleable ElGamal encryption scheme by combining Schnorr's signature scheme 25 with ElGamal encryption scheme under some cryptographic assumption in the random oracle model. Jakobsson used the non-malleable ElGamal encryption scheme in his MIX net for users' encryption to prevent the repeated ciphertext attack 13 . (For a detailed study of the security consult 26.) We also use this scheme in our MIX net of Sect. 4

\section{An Attack for Jakobsson's Practical MIX}

In this section, we show how to break the Jakobsson's MIX net of Eurocrypt'98 I3, which was believed to be $t$-resilient and very efficient. 
Jakobsson first showed that a MIX net is obtained by using MIXEXP which takes a list of items $\bar{\mu}=\left(c_{1}, \ldots, c_{N}\right)$ and robustly computes a permutation $\left(c_{1}^{\delta}, \ldots, c_{N}^{\delta}\right)$. To avoid cut and choose methods, Jakobsson 13 developed a subprotocol in which each MIX server proves that the product of his input elements and the product of his output elements satisfy a certain relation. However, this does not imply proving that each MIX server behaved correctly even if the subprotocol is combined with his other subprotocols. We show an attack such that all the output elements of a MIX server can be affected in a proper way. We also exploit a homomorphic property of his scheme to attack it.

His MIX net is not robust if the MIXEXP is not robust. Therefore, the details of MIXEXP are given in Sect. B.J Our attack is given in Sect. 3.2

If the reader is not interested or is already familiar with his scheme, he can go directly to Sect. 32

\subsection{Structure of the Scheme Attacked 13}

Let

$$
\Delta_{j}=g^{\delta_{j}}
$$

be the public information of a MIX server $j$, where $\delta_{j}$ is his secret. Define

$$
\delta \triangleq \prod_{j \in Q} \delta_{j},
$$

where $Q$ denotes a quorum. MIXEXP takes a list of items $\bar{\mu}=\left(c_{1}, \ldots, c_{N}\right)$ and robustly computes a permutation $\left(c_{1}^{\delta}, \ldots, c_{N}^{\delta}\right)$.

Jakobsson then showed an efficient implementation of MIXEXP. It consists of four protocols, Blinding I, Blinding II, Unblinding I and Unblinding II. For simplicity, let $Q=\{1,2, \cdots, t+1\}$. For a list $\bar{\xi}=\left(d_{1}, \ldots, d_{N}\right)$ and $e \in Z_{q}$, define

$$
\bar{\xi}^{e} \triangleq\left(d_{1}^{e}, \ldots, d_{N}^{e}\right)
$$

Let $\kappa$ be a security parameter.

Blinding I: (see Fig. 1) For $1 \leq \lambda \leq \kappa$,

1. MIX server 1 chooses a random number $\rho_{I \lambda_{1}}$ and a random permutation $\pi_{I \lambda_{1}}$. He then computes

$$
\pi_{I \lambda_{1}}\left(c_{1}^{\rho_{I \lambda_{1}}}, c_{2}^{\rho_{I \lambda_{1}}}, \cdots, c_{N}^{\rho_{I \lambda_{1}}}\right) .
$$

2. MIX server 2 chooses a random number $\rho_{I \lambda_{2}}$ and a random permutation $\pi_{I \lambda_{2}}$. He then computes

$$
\pi_{I \lambda_{2}} \circ \pi_{I \lambda_{1}}\left(c_{1}^{\rho_{I \lambda_{1}} \rho_{I \lambda_{2}}}, c_{2}^{\rho_{I \lambda_{1}} \rho_{I \lambda_{2}}}, \cdots, c_{N}^{\rho_{I \lambda_{1}} \rho_{I \lambda_{2}}}\right),
$$

and so on. 


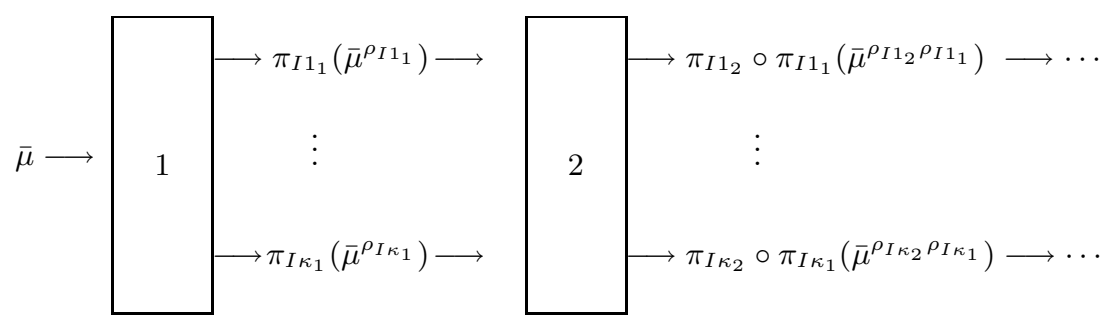

Fig. 1. Blinding I

The final output (in Blinding I) from MIX server $t+1$ is:

$$
\bar{\mu}_{I \lambda} \triangleq \pi_{I \lambda}\left(c_{1}^{\rho_{I \lambda}}, c_{2}^{\rho_{I \lambda}}, \cdots, c_{N}^{\rho_{I \lambda}}\right),
$$

where

$$
\pi_{I \lambda} \triangleq \prod_{j \in Q} \pi_{I \lambda j}, \quad \rho_{I \lambda} \triangleq \prod_{j \in Q} \rho_{I \lambda j} .
$$

That is, MIXEXP outputs $\bar{\mu}_{I 1}, \cdots, \bar{\mu}_{I \kappa}$ on input $\bar{\mu}$ in Blinding I.

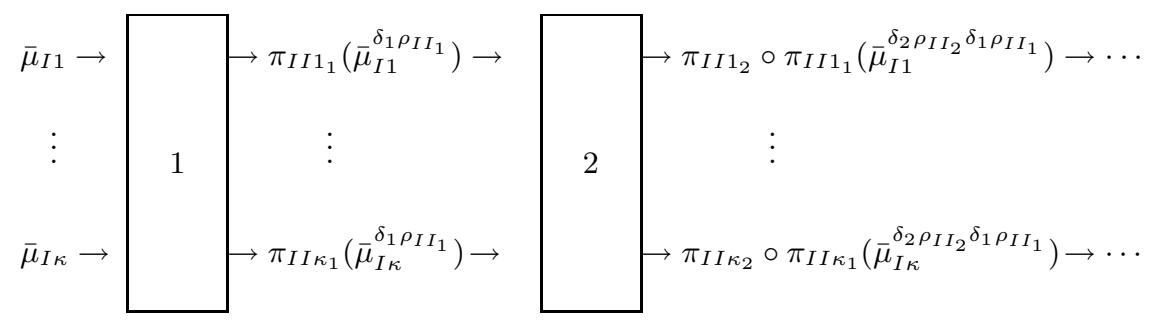

Fig. 2. Blinding II

Blinding II: (see Fig. 2) For $1 \leq \lambda \leq \kappa$,

1. MIX server 1 chooses a random number $\rho_{I I_{1}}$ and a random permutation $\pi_{I I \lambda_{1}}$. He then computes

$$
\begin{gathered}
\pi_{I I \lambda_{1}}\left(\bar{\mu}_{I \lambda}^{\delta_{1} \rho_{I I_{1}}}\right)=\pi_{I I \lambda_{1}} \circ \pi_{I \lambda}\left(\left(c_{1}^{\rho_{I \lambda}}\right)^{\delta_{1} \rho_{I I_{1}}},\left(c_{2}^{\rho_{I \lambda}}\right)^{\delta_{1} \rho_{I I_{1}}},\right. \\
\left.\cdots,\left(c_{N}^{\rho_{I \lambda}}\right)^{\delta_{1} \rho_{I I_{1}}}\right)
\end{gathered}
$$

from $\bar{\mu}_{I \lambda}$. Note that $\rho_{I I_{1}}$ is independent of $\lambda$ while $\rho_{I \lambda_{1}}$ depends on $\lambda$. 
2. MIX server 2 chooses a random number $\rho_{I I_{2}}$ and a random permutation $\pi_{I I \lambda_{2}}$. He then computes

$$
\begin{gathered}
\pi_{I I \lambda_{2}} \circ \pi_{I I \lambda_{1}} \circ \pi_{I \lambda}\left(\left(c_{1}^{\rho_{I \lambda}}\right)^{\delta_{1} \rho_{I I_{1}} \delta_{2} \rho_{I I_{2}}},\left(c_{2}^{\rho_{I \lambda}}\right)^{\delta_{1} \rho_{I I_{1}} \delta_{2} \rho_{I I_{2}}},\right. \\
\left.\cdots,\left(c_{N}^{\rho_{I \lambda}}\right)^{\delta_{1} \rho_{I I_{1}} \delta_{2} \rho_{I I_{2}}}\right)
\end{gathered}
$$

and so on.

The final output (in Blinding II) of MIX server $t+1$ is:

$$
\bar{\sigma}_{I I \lambda} \triangleq \pi_{I I \lambda} \circ \pi_{I \lambda}\left(c_{1}^{\rho_{I \lambda} \delta \rho_{I I}}, c_{2}^{\rho_{I \lambda} \delta \rho_{I I}}, \cdots, c_{N}^{\rho_{I \lambda} \delta \rho_{I I}}\right),
$$

where $\delta$ is defined in eq. 2 and

$$
\pi_{I I \lambda} \triangleq \prod_{j \in Q} \pi_{I I \lambda j}, \quad \rho_{I I} \triangleq \prod_{j \in Q} \rho_{I I j} .
$$

That is, MIXEXP outputs $\bar{\sigma}_{I I 1}, \cdots, \bar{\sigma}_{I I \kappa}$ on input $\bar{\mu}_{I 1}, \cdots, \bar{\mu}_{I \kappa}$ in Blinding II.

From eq. 14, we see that

$$
\bar{\sigma}_{I I \lambda}^{1 / \rho_{I \lambda}}=\pi_{I I \lambda} \circ \pi_{I \lambda}\left(c_{1}^{\delta \rho_{I I}}, \ldots, c_{N}^{\delta \rho_{I I}}\right) .
$$

Note that $\left(c_{1}^{\delta \rho_{I I}}, \ldots, c_{N}^{\delta \rho_{I I}}\right)$ of the right hand side is independent of $\lambda$. Therefore, $\bar{\sigma}_{I I \lambda}^{1 / \rho_{I \lambda}}$ must be equal for $1 \leq \lambda \leq \kappa$ if each list $\pi_{I I \lambda} \circ \pi_{I \lambda}\left(c_{1}^{\delta \rho_{I I}}, \ldots, c_{N}^{\delta \rho_{I I}}\right)$ is sorted. Unblinding I, based on this observation, is described as follows.

\section{Unblinding I:}

1. Each MIX server $j$ publishes $\left\{\rho_{I \lambda j}\right\}$ for $1 \leq \lambda \leq \kappa$.

2. Each MIX server computes $\rho_{I \lambda}=\prod_{j \in Q} \rho_{I \lambda j}$ and

$$
\bar{\sigma}_{I \lambda} \triangleq \bar{\sigma}_{I I \lambda}^{1 / \rho_{I \lambda}}=\pi_{I I \lambda} \circ \pi_{I \lambda}\left(c_{1}^{\delta \rho_{I I}}, \ldots, c_{N}^{\delta \rho_{I I}}\right)
$$

for $1 \leq \lambda \leq \kappa$.

3. The lists $\bar{\sigma}_{I \lambda}$ with $1 \leq \lambda \leq \kappa$ are sorted and compared. If they are all equal, and no element is zero, then the result is labeled valid, otherwise invalid.

Next in Blinding II for $\lambda=1$, let $M_{j}$ denote the product (modulo $p$ ) of all the elements constituting the input to MIX server $j$. Similarly, $S_{j}$ denotes the product of all the output elements of MIX server $j$. Then it must hold that

$$
S_{j}=M_{j}^{\delta_{j} \rho_{I I_{j}}}
$$

On the other hand, from eq. II , we have $\Delta_{j}^{\rho_{I I}}=g^{\delta_{j} \rho_{I I_{j}}}$. Therefore, it holds that

$$
S_{j}=M_{j}^{z} \text { and } \Delta_{j}^{\rho_{I I_{j}}}=g^{z}
$$

for $z=\delta_{j} \rho_{I I_{j}}$. Unblinding II, based on this observation, is described as follows. 
Unblinding II: (for valid results only)

1. The MIX servers publish $\left\{\pi_{I 1 j}\right\}_{j \in Q}$.

2. The computation of $\bar{\mu}_{I 1}$ in "Blinding I" is verified.

3. The MIX servers publish $\left\{\rho_{I I j}\right\}_{j \in Q}$.

4. Each MIX server $j$ proves that

$$
S_{j}=M_{j}^{z} \text { and } \Delta_{j}^{\rho_{I I_{j}}}=g^{z} .
$$

holds for some $z$ by using one of the methods of 725 .

5. The MIX servers compute $\bar{\sigma}_{1}=\bar{\sigma}_{I 1}^{1 / \rho_{I I}}$, and output $\bar{\sigma}_{1}$. Note that $\bar{\sigma}_{1}$ is a permutation of $\left(c_{1}^{\delta}, \ldots, c_{N}^{\delta}\right)$ from eq. 15 .

Jakobsson claims that the final output $\bar{\sigma}_{1}$ is a permutation of $\left(c_{1}^{\delta}, \ldots, c_{N}^{\delta}\right)$ if the above protocol (MIXEXP) ends successfully.

\subsection{Our Attack}

We show that Jakobsson's MIXEXP is not robust. This means that his MIX net is not robust. Our attack succeeds if at least one MIX server is malicious.

We exploit a homomorphic property. A dishonest MIX server will first multiply the received inputs. The data is then organized to prevent detection. We now describe the details.

For simplicity, suppose that the last MIX server $t+1$ of $Q$ is malicious. In Blinding II, let her input be $\left(d_{\lambda 1}, \ldots, d_{\lambda N}\right)$ for $1 \leq \lambda \leq \kappa$. Let

$$
X_{\lambda} \triangleq d_{\lambda 1} * \cdots * d_{\lambda N}
$$

In our attack, she first chooses random numbers $\alpha_{1}, \cdots, \alpha_{N}$ such that

$$
\alpha_{1}+\alpha_{2}+\cdots \alpha_{N}=1 \bmod q
$$

Next she outputs

$$
\tilde{\sigma}_{I I \lambda}=\left(X_{\lambda}^{\alpha_{1} \delta_{t+1} \rho_{I I} I_{t+1}}, X_{\lambda}^{\alpha_{2} \delta_{t+1} \rho_{I I}}{ }, \ldots, X_{\lambda}^{\alpha_{N} \delta_{t+1} \rho_{I I} I_{t+1}}\right)
$$

for $1 \leq \lambda \leq \kappa$.

We next show that the MIXEXP ends successfully and our cheating is not detected.

Theorem 1. The check of Unblinding I is satisfied.

Proof: In Blinding II, the output of MIX server $t+1$ is $\bar{\sigma}_{I I \lambda}$ of eq. (4) if she is honest. Therefore, her input must be

$$
\begin{aligned}
\left(d_{\lambda_{1}}, \ldots, d_{\lambda_{N}}\right) & =\pi_{I I \lambda_{t+1}}^{-1}\left(\bar{\sigma}_{I I \lambda}^{1 / \delta_{t+1} \rho_{I I} I_{t+1}}\right) \\
& =\theta_{\lambda}\left(c_{1}^{\rho_{I \lambda} \delta \rho_{I I} / \delta_{t+1} \rho_{I I} t+1}, \ldots, c_{N}^{\rho_{I \lambda} \delta \rho_{I I} / \delta_{t+1} \rho_{I I} t+1}\right)
\end{aligned}
$$


for some permutation $\theta_{\lambda}$ for $1 \leq \lambda \leq \kappa$. Therefore,

$$
X_{\lambda}=d_{\lambda_{1}} * \cdots * d_{\lambda_{N}}=\left(c_{1} * \cdots * c_{N}\right)^{\rho_{I \lambda} \delta \rho_{I I} / \delta_{t+1} \rho_{I I_{t+1}}}=Z^{\rho_{I \lambda} \delta \rho_{I I} / \delta_{t+1} \rho_{I I_{t+1}}},
$$

where $Z \triangleq c_{1} * \cdots * c_{N}$. Then eq. 8 is written as

$$
\tilde{\sigma}_{I I \lambda}=\left(Z^{\alpha_{1} \rho_{I \lambda} \delta \rho_{I I}}, \ldots, Z^{\alpha_{N} \rho_{I \lambda} \delta \rho_{I I}}\right) .
$$

Finally, at Step 2 of Unblinding I, each MIX server computes

$$
\tilde{\sigma}_{I \lambda}=\tilde{\sigma}_{I I \lambda}^{1 / \rho_{I \lambda}}=\left(Z^{\alpha_{1} \delta \rho_{I I}}, \ldots, Z^{\alpha_{N} \delta \rho_{I I}}\right)
$$

for $1 \leq \lambda \leq \kappa$. Note that $\left(Z^{\alpha_{1} \delta \rho_{I I}}, \ldots, Z^{\alpha_{N} \delta \rho_{I I}}\right)$ is independent of $\lambda$. Therefore, we see that $\tilde{\sigma}_{I 1}=\tilde{\sigma}_{I 2}=\cdots=\tilde{\sigma}_{I \kappa}$. This means that the check of Unblinding I is satisfied.

Theorem 2. The check of Unblinding II is satisfied.

Proof: Note that $M_{t+1}=X_{1}$ and $S_{t+1}$ is the product of all the elements of eq. 8 for $\lambda=1$. Therefore, we have

$$
\begin{aligned}
S_{t+1} & =X_{1}^{\alpha_{1} \delta_{t+1} \rho_{I I_{t+1}}} * \cdots * X_{1}^{\alpha_{N} \delta_{t+1} \rho_{I I I+1}}=\left(X_{1}^{\delta_{t+1} \rho_{I I} I_{t+1}}\right)^{\alpha_{1}+\cdots+\alpha_{N}} \\
& =\left(M_{t+1}\right)^{\delta_{t+1} \rho_{I I_{t+1}}} .
\end{aligned}
$$

Thus, eq. $\mathbf{b}$ is satisfied. Hence, eq. $\mathbf{\square}$ is satisfied for some $z$.

Finally, from eq. 9 and Step 5 of Unblinding II, the output of the MIXEXP becomes as follows.

$$
\begin{aligned}
\tilde{\sigma}_{1} & =\tilde{\sigma}_{I 1}^{1 / \rho_{I I}} \\
& =\left(Z^{\alpha_{1} \delta}, \cdots, Z^{\alpha_{N} \delta}\right) \\
& =\left(\left(c_{1} * \cdots * c_{N}\right)^{\alpha_{1} \delta}, \cdots,\left(c_{1} * \cdots * c_{N}\right)^{\alpha_{N} \delta}\right) .
\end{aligned}
$$

This is clearly different from a permutation of $\left(c_{1}^{\delta}, \ldots, c_{N}^{\delta}\right)$. (See Step 5 of Unblinding II.) Therefore, the MIXEXP does not compute the correct output without being detected.

\section{Proposed MIX Net}

In this section, we show an efficient $t$-resilient MIX net by using a certain combinatorial structure over the set of MIX servers.

In Sect. 1 l. we introduce two new concepts, existential-honesty and limitedopen-verification. They will be useful for distributed computation in general. We also define a combinatorial structure which guarantees that our scheme is $t$-resilient.

Our scheme is given in Sect. 4.2 and Sect. 4.3 We further show an efficient construction of the combinatorial structure by using covering 29 in Sect. 4.4 Covering has recently been used in another cryptographic application: robust secret sharing by Rees et al. 2.3 . 


\subsection{Existential-Honesty and Limited-Open-Verification}

A set system is a pair $(X, \mathcal{B})$, where $X \triangleq\{1,2, \ldots, v\}$ and $\mathcal{B}$ is a collection of blocks $B_{i} \subset X$ with $i=1,2, \ldots, b$. First, we define $(v, b, t)$-verifiers set systems.

Definition 1. We say that $(X, \mathcal{B})$ is a $(v, b, t)$-verifiers set system if

1. $\left|B_{i}\right|=t+1$ for $i=1,2, \ldots, b$ and

2. for any subset $F \subset X$ with $|F| \leq t$, there exists a $B_{i} \in \mathcal{B}$ such that $F \cap B_{i}=\emptyset$.

Let $(X, \mathcal{B})$ be a $(v, b, t)$-verifiers set system. We identify $X$ with the set of MIX servers. Therefore, $B_{i}$ is a subset of MIX servers of size $t+1$. We choose $P_{i} \in B_{i}$ arbitrarily for $1 \leq i \leq b . P_{i}$ is called a prover. The other MIX servers of $B_{i}$ are called verifiers.

We introduce two new concepts in this paper,

- Existential-honesty and

- $t$-open-verification

which we now describe.

Existential honesty follows from Definition II Although, existential honesty is not limited to applications in the MIX context, and may be useful in other distributed computation, we focus on its MIX application. In each block one designated party will mix the ciphertexts. As long as one block of MIX servers is free of dishonest machines, the goal of mixing has been achieved. Now we do not know which block satisfies this property. However, Definition II guarantees that there always exists one block of honest parties. So, we let each block mix the ciphertexts (i.e. the designated party of that block). What do we do when the designated party of a block $B_{j}$ is dishonest? Since a block has $t+1$ parties, it must be detected. Indeed, there are at most $t$ dishonest parties. If it is detected, then we ignore the output and proceed with the output of block $B_{j-1}$ (or an even earlier one if the designated party in $B_{j-1}$ was dishonest). Now, what happens when one of the verifiers falsely accuses the mixing party of having been dishonest. Then we know that this block is not free of dishonest parties, and therefore the block under consideration is not the one of Definition Il so we can just ignore the output of the block. In other words, we do not have to decide whether the one who mixed it was honest or not.

We now explain t-open-verification. In many secure distributed computation protocols zero-knowledge is used to prove that the computation was done correctly. In our approach we do not need zero-knowledge, the prover will reveal the secrets he used. However, he will only do this to $t$ parties. The existential honesty guarantees that all parties in at least one of the blocks of MIX servers will all be honest. So, the prover can reveal the secret he used. This speeds up the verification dramatically.

We now formally describe the scheme in full detail. 


\subsection{Initialization}

Let $y\left(=g^{x} \bmod p\right)$ be a public key of the ElGamal scheme as shown in Sect. 2.2 We assume that the secret key $x$ is distributed among $v$ MIX servers by using Shamir's $(t+1, v)$ secret sharing scheme. Actually, we use a robust $(t+1, v)$ threshold ElGamal decryption scheme. (See the end of the next subsection for more details.)

1. Each user $i$ computes a ciphertext $c_{i}=\left(a_{i}, b_{i}, a u x_{i}, s_{i} g_{i}\right)$ by the non-malleable ElGamal encryption scheme 28 as shown in Sect. 2.3 That is, $\left(a_{i}, b_{i}\right)=$ $\left(g^{\gamma_{i}}, m_{i} y^{\gamma_{i}}\right)$ is the ciphertext of the usual ElGamal scheme, aux $x_{i}$ is the auxiliary information and $s i g_{i}$ is the Schnorr's signature of $\left(a_{i}, b_{i}, a u x_{i}\right)$ such that $a_{i}$ is a public key and $\gamma_{i}$ is the secret key.

2. Each user $i$ posts his ciphertext $c_{i}$ to the bulletin board.

3. $c_{i}$ is discarded if the signature is not valid.

\subsection{Main Protocol}

We assume that all MIX servers of $B_{i}$ share a common key $e_{i}$ for $1 \leq i \leq b$. We extract $\left(a_{i}, b_{i}\right)$ from a valid ciphertext $c_{i}$. Let

$$
A_{0}=\left(\left(a_{1}, b_{1}\right), \ldots,\left(a_{N}, b_{N}\right)\right) .
$$

We wish to produce a random permutation of the list $\left(m_{1}, \ldots, m_{N}\right)$, where $m_{i}=b_{i} / a_{i}^{x}$ is the plaintext of $\left(a_{i}, b_{i}\right)$. A prover of a block $B_{i}$ first publishes $A_{1}$ which is a randomly permuted list of reencrypted ciphertexts of $A_{0}$. He then privately broadcasts the secret random string $R_{i}$ he used to the verifiers in the same block $B_{i}$. Each verifier of $B_{i}$ checks the validity of $A_{0}$ by using $R_{i}$.

For $j=1, \ldots, b$, do:

Step 1. Let

$$
A_{0}=\left(\left(\hat{a}_{1}, \hat{b}_{1}\right), \ldots,\left(\hat{a}_{N}, \hat{b}_{N}\right)\right) .
$$

The prover $P_{j}$ of block $B_{j}$ chooses random numbers $s_{1}, \ldots, s_{N}$ and a random permutation $\pi_{j}$. She computes

$$
A_{1}=\pi_{j}\left(\left(\hat{a}_{1} g^{s_{1}}, \hat{b}_{1} y^{s_{1}}\right), \ldots,\left(\hat{a}_{N} g^{s_{N}}, \hat{b}_{N} y^{s_{N}}\right)\right) .
$$

and then publishes $A_{1}$. ( $A_{1}$ is commonly used for all the verifiers of $B_{j}$.)

Step 2. $P_{j}$ encrypts $s_{1}, \ldots, s_{N}$ and $\pi_{j}$ by the key $e_{j}$ of block $B_{j}$. Then $P_{j}$ publishes these ciphertexts. $\left(P_{j}\right.$ is broadcasting $s_{1}, \ldots, s_{N}$ and $\pi_{j}$ secretly to all the verifiers of $B_{j}$.)

Step 3. Each verifier of block $B_{j}$ decrypts the above ciphertexts and checks whether $A_{1}$ is computed correctly by using $s_{1}, \ldots, s_{N}$ and $\pi_{j}$. He outputs "ACCEPT" if $A_{1}$ is computed correctly and "REJECT" otherwise.

Step 4. If some verifier of block $B_{j}$ outputs "REJECT", then $A_{1}$ is ignored. Otherwise, let $A_{0}:=A_{1}$. 
Let the final result be $A_{0}=\left(\left(\hat{c}_{1}, \hat{d}_{1}\right), \ldots,\left(\hat{c}_{N}, \hat{d}_{N}\right)\right)$.

Next any $(t+1)$ MIX servers decrypt each $\left(\hat{c}_{i}, \hat{d}_{i}\right)$ by using a robust $(t+1, v)$ threshold ElGamal decryption scheme. Finally, we obtain a random permutation of the list $\left(m_{1}, \ldots, m_{N}\right)$.

Gennaro et al. showed a robust threshold RSA signature scheme in 12. A robust $(t+1, v)$ threshold ElGamal decryption scheme is easily obtained by applying their technique to ElGamal decryption.

\subsection{Construction of the Set System}

Let $v=(t+1)^{2}, b=t+1, X=\left\{1,2, \cdots,(t+1)^{2}\right\}$ and $B_{i}=\{(i-1)(t+$ $1)+1, \cdots, i(t+1)\}$ for $1 \leq i \leq b$. Then it is easy to see that $(X, \mathcal{B})$ is a $\left((t+1)^{2}, t+1, t\right)$-verifiers set system.

We next show a more efficient $(v, b, t)$-verifiers set system. A set $\operatorname{system}(X, \mathcal{B})$ is called a $(v, k, t)$-covering if $z$

1. $\left|B_{i}\right|=k$ for $1 \leq i \leq b$ and

2. every $t$-subset of $X$ is included in at least one block.

From 17, we have the following proposition. (We learned about proposition II from 2.3.)

Proposition 1. Suppose that $k=$ even and

$$
\begin{aligned}
3 & \leq s \leq \frac{t+3}{2} \\
k\left(t-\frac{s-3}{2}\right) & \leq v<k\left(t-\frac{s-4}{2}\right) .
\end{aligned}
$$

Then there exists $a(v, v-k, t)$-covering such that $b=t+s$. Further, each element $a \in X$ is included in at most two blocks.

See 1723 for the construction.

We next borrow the following lemma from 23 in which the lemma was used for robust secret sharing schemes. The proof will be clear.

Lemma 1. $(X, \mathcal{B})$ is a $(v, b, t)$-verifiers set system if and only if the set system $\left(X, \mathcal{B}^{c}\right)$ is a $(v, v-t-1, t)$-covering, where $\mathcal{B}^{c} \triangleq\left\{X \backslash B_{i} \mid B_{i} \in \mathcal{B}\right\}$.

Then we obtain a $(v, b, t)$-verifiers set system as follows.

Corollary 1. For $t=$ odd, there exists $a(v, b, t)$-verifiers set system such that

$$
v=\frac{3}{4}(t+1)^{2} \text { and } b=\frac{3}{2}(t+1) .
$$

Further, each element $a \in X$ is included in at most two blocks. 
Proof: In Proposition II let $k=t+1$ and $s=(t+3) / 2$.

The fact that each MIX server is included in at most two blocks is primordial to understand the efficiency analysis described further on.

We show a small example of Corollary ll Let $t=3, b=6, v=12$ and

$$
\begin{gathered}
B_{1}=(1,2,3,4), B_{2}=(3,4,5,6), B_{3}=(5,6,1,2), \\
B_{4}=(7,8,9,10), B_{5}=(9,10,11,12), B_{6}=(11,12,7,8)
\end{gathered}
$$

Then it is easy to see that this is a $(12,6,3)$-verifiers set system which satisfies Corollary II

\subsection{Efficiency}

In the $(v, b, t)$-verifiers set system of Corollary 1 each MIX server is included in at most two blocks. Therefore, each MIX server acts as a prover at most twice and acts as a verifier at most twice.

In Step 1 and Step 2, each prover computes $A_{1}$ and encrypts $s_{1}, \cdots, s_{N}$ and $\pi_{j}$. This computation cost is $O(N)$. He publishes $A_{1}$ and the ciphertexts of $s_{1}, \cdots, s_{N}$ and $\pi_{j}$. This communication cost is $O(N)$. Therefore, the total cost of the prover is $O(N)$. In Step 3, each verifier decrypts the ciphertexts of $s_{1}, \cdots, s_{N}$, $\pi_{j}$ and checks the validity of $A_{1}$. This computation cost is $O(N)$. He publishes "ACCEPT" or "REJECT". This communication cost is $O(1)$. Therefore, the total cost of the verifier is $O(N)$. In the end, the total cost of each MIX server is $O(N)$.

An alternative method to compute the computation cost per user is to analyze the total cost and then divide by the total number of MIX servers. One needs then to take into account that the number of MIX servers is $O\left(t^{2}\right)$, compared to $O(t)$ in previous work.

\section{Security of the Protocol}

\section{$5.1 \quad$ Verifiability}

Suppose that the prover $P_{j}$ of $B_{j}$ is malicious and $A_{1}$ is not correctly computed. Then there exists at least one honest verifier in $B_{j}$ because $\left|B_{j}\right|=t+1$ and there exist at most $t$ malicious MIX servers. The honest verifier outputs "REJECT" at Step 3. Therefore, $A_{1}$ is ignored at Step 4.

\subsection{Robustness}

For any $t$ malicious MIX servers, there exists at least one $B_{i}$ in which all MIX server are honest from Def II This $B_{i}$ computes $A_{1}$ correctly. On the other hand, any invalid $A_{1}$ is ignored from the verifiability. Therefore, our protocol outputs a random permutation of $\left(m_{1}, \ldots, m_{N}\right)$ correctly even if there are at most $t$ malicious MIX servers. 


\subsection{Privacy (Sketch)}

The ElGamal based encryption scheme of 28 is known to be non-malleable under adaptive chosen ciphertext attack. Let $c_{1}, c_{2}$ be two ciphertexts and $m_{1}, m_{2}$ be the plaintexts. Then by using the result of 4 , we can show that there exists no probabilistic polynomial time (p.p.t.) Turing machine $D$ (distinguisher) which can distinguish $\left(c_{1}, c_{2}, m_{1}, m_{2}\right)$ and $\left(c_{1}, c_{2}, m_{2}, m_{1}\right)$ with meaningful probability. This is the minimum requirement that any MIX net of this type must satisfy. We also assume that it satisfies plaintext awareness 5 which means that no p.p.t. adversary can create a ciphertext $c$ without knowing its underlying plaintext $m$.

Now consider an adversary $M_{0}$ who can control at most $t$ MIX servers and at most $N-2$ out of the $N$ users posting encrypted messages. It is the goal of the adversary $M_{0}$ to match each one of the two plaintexts $m_{1}, m_{2}$ to their corresponding ciphertexts $c_{1}, c_{2}$ that he does not control. In other words, $M_{0}$ wishes to distinguish $\left(c_{1}, c_{2}, m_{1}, m_{2}\right)$ and $\left(c_{1}, c_{2}, m_{2}, m_{1}\right)$.

Suppose that there exists a p.p.t. adversary $M_{0}$ who can distinguish $\left(c_{1}, c_{2}\right.$, $\left.m_{1}, m_{2}\right)$ and $\left(c_{1}, c_{2}, m_{2}, m_{1}\right)$ with meaningful probability. For simplicity, suppose that $M_{0}$ controls users $3, \cdots, N$.

We will show a distinguisher $D$. The input to $D$ is $\left(c_{1}, c_{2}, z_{1}, z_{2}\right)$, where $\left(z_{1}, z_{2}\right)=\left(m_{1}, m_{2}\right)$ or $\left(m_{2}, m_{1}\right) . D$ first gives $c_{1}, c_{2}$ to $M_{0}$ and runs the users part of $M_{0}$. Then $M_{0}$ outputs $c_{3}, \cdots, c_{N}$. From the plaintext awareness assumption, $M_{0}$ knows the plaintexts $m_{3}, \cdots, m_{N}$ for $c_{3}, \cdots, c_{N}$. Therefore, $D$ knows the set of $\left\{m_{1}, m_{2}, m_{3}, \cdots, m_{N}\right\}$.

$D$ next runs the main body of our protocol in such a way that $D$ simulates the part of honest MIX servers faithfully and uses $M_{0}$ for the part of malicious MIX servers. Let the output of the main body be $A_{0}=\left(\left(\hat{c}_{1}, \hat{d}_{1}\right), \ldots,\left(\hat{c}_{N}, \hat{d}_{N}\right)\right)$. Note that $A_{0}$ is a random permutation of randomized ciphertexts $c_{1}, \cdots, c_{N}$ from Sect. 5.2

Let $\pi$ be a random permutation. Let $\hat{m}_{i}$ denote the plaintext of $\left(\hat{c}_{i}, \hat{d}_{i}\right)$ for $1 \leq i \leq N$. Then we can show that $M_{0}$ cannot distinguish $\left(\hat{c}_{i}, \hat{d}_{i}, \hat{m}_{i}\right)$ and $\left(\hat{c}_{i}, \hat{d}_{i}, m_{\pi(i)}\right)$ under the decision Diffie-Hellman assumption. $D$ finally generates a view of $M_{0}$ for the robust $(t+1, n)$ threshold ElGamal decryption scheme with $\left(\hat{c}_{i}, \hat{d}_{i}, m_{\pi(i)}\right)$ by using the technique of 812 for $1 \leq i \leq N$.

Then $M_{0}$ can distinguish $\left(c_{1}, c_{2}, m_{1}, m_{2}\right)$ and $\left(c_{1}, c_{2}, m_{2}, m_{1}\right)$ with meaningful probability from our assumption on $M_{0}$. Hence, $D$ can distinguish $\left(c_{1}, c_{2}, m_{1}, m_{2}\right)$ and $\left(c_{1}, c_{2}, m_{2}, m_{1}\right)$ with meaningful probability. However, this is a contradiction.

\section{Open Problems}

This paper introduces several open problems, in particular:

- whether the new tools of existential-honesty and limited-open-verification can be used in other secure distributed computation. 
- whether there are other choices of $v$. Indeed, when $t$ is large the required number of MIX servers only grows quadratic. Although this is reasonable for a theoretician, from a practical viewpoint, the question is worth addressing.

- is $O(N)$ the minimum required effort per MIX server while maintaining $t$ privacy, $t$-verifiability, and $t$-robustness, in a network with $O\left(t^{2}\right)$ servers.

\section{Acknowledgement}

The authors thank Prof. Stinson for providing in August 1999 the second author of the paper with a preliminary copy of their Rees et al. paper 23. The authors are grateful for the many comments received from the referee and from Dr. Jakobsson who shepherded the rewriting of the paper. Many of these comments have significantly contributed to increase the readability of the text.

\section{References}

1. M. Abe, "Universally verifiable mix-net with verification work independent of the number of mix-centers," Eurocrypt '98, pp. 437-447.

2. M. Abe, "A mix-network on permutation networks," ISEC Technical report 99-10 (in Japanese) (May, 1999)

3. M. Abe, "Mix-networks on permutation networks," Asiacrypt '99, pp. 258-273.

4. M. Bellare, A. Desai, D. Poincheval, P. Rogaway, "Relations among notions of security for public key encryption schemes," Crypto '98, pp. 26-45.

5. M. Bellare, P. Rogaway, "Optimal asymmetric encryption - How to encrypt with RSA," Eurocrypt '94, pp. 92-111.

6. D. Chaum, "Untraceable electronic mail, return addresses, and digital pseudonyms," Communications of the ACM, Vol. 24, 1981, pp. 84-88.

7. D. Chaum, H. Van Antwerpen, "Undeniable signatures," Crypto '89, pp. 212-216.

8. Y. Desmedt, Y. Frankel, "Threshold cryptosystems," Crypto '89, pp. 307-315.

9. D. Dolev, C. Dwork, M. Naor, "Non-malleable cryptography," STOC '91, pp. 542552.

10. T. ElGamal, "A public-key cryptosystem and a signature scheme based on discrete logarithms," Crypto '84, pp. 10-18.

11. A. Fujioka, T. Okamoto, K. Ohta, "A practical secret voting scheme for large scale elections," Auscrypt '92, pp. 244-251.

12. R. Gennaro, S. Jarecki, H. Krawczyk, T. Rabin, "Robust and efficient sharing of RSA functions," Crypto '96, pp. 157-172.

13. M. Jakobsson, "A practical MIX," Eurocrypt '98, pp. 448-461.

14. M. Jakobsson, D. M'Raihi, "Mix-based electronic payments," SAC'98, pp. 157-173.

15. M. Jakobsson, "Flash mixing," PODC'99, pp. 83-89.

16. M. Jakobsson, A. Juels "Millimix: Mixing in small batches," DIMACS Technical report 99-33 (June 1999)

17. W. H. Mills, "Covering design I: coverings by a small number of subsets," Ars Combin. 8, (1979), pp. 199-315.

18. W. Ogata, K. Kurosawa, K. Sako, K. Takatani, "Fault tolerant anonymous channel," ICICS '97, pp. 440-444.

19. C. Park, K. Itoh, K. Kurosawa, "All/nothing election scheme and anonymous channel," Eurocrypt '93, pp. 248-259. 
20. T. P. Pedersen, "A threshold cryptosystem without a trusted party," Eurocrypt '91, pp. 522-526.

21. B. Pfitzmann, A. Pfitzmann. "How to break the direct RSA-implementation of MIXes," Eurocrypt '89, pp. 373-381.

22. D. Pointcheval, J. Stern, "Security proofs for signature schemes," Eurocrypt '96, pp. 387-398.

23. R. Rees, D. R. Stinson, R. Wei, G. H. J. van Rees, "An application of covering designs: Determining the maximum consistent set of shares in a threshold scheme," Ars Combin. 531 (1999), pp. 225-237.

24. K. Sako, J. Kilian, "Receipt-free mix-type voting scheme," Eurocrypt '95, pp. 393403.

25. C. P. Schnorr, "Efficient signature generation for smart cards," Crypto '89, pp. 239252.

26. C. P. Schnorr, M. Jakobsson, "Security of discrete log cryptosystems in the random oracle + generic model," http://www.bell-labs.com/user/markusj/

27. A. Shamir, "How to share a secret," Communications of the ACM, Vol. 22, 1979, pp. $612-613$

28. Y. Tsiounis, M. Yung, "On the security of ElGamal based encryption," PKC'98, pp. $117-134$.

29. Edited by C. J. Colbourn and J. H. Dinitz, Handbook of Combinatorial Design, CRC Press (1996) 